\title{
Analysis of the Relation between Biological Classification Ability and Cortisol-hormonal Change of Middle School Students
}

\author{
Ye-Jun Bae · II-Sun Lee · Jung-Ho Byeon · Yong-Ju Kwon* \\ Korea National University of Education
}

\begin{abstract}
The purpose of this study is to investigate the relation between the classification ability quotient and cortisol-hormonal change of middle school students. Thirty-three students, second graders in middle school, performed the classification task that can be an indicator of students' classification ability. And then amount of the secreted hormone was analyzed during task performance. The study results were as follows: First, the classification methods of students mostly utilized visual, qualitative. Their classification patterns for each subject were static, partial, and non-comparative. Second, the amount of stress-hormone was secreted from students during the experiment decreased in overall after the free classification. It seemed that student-centered activity relieved stress. Third, the classification ability quotient turned out to be significantly correlated to the stress hormone, which means that there was a close relationship between classification ability and stress level. It was also considered that stress had a positive effect on the improvement of classification ability. This study provided physiologically more accurate information on the stress increased in the learning process than other conventional studies based on reports or interviews. Finally, researchers could recognize the effect of stress in the cognitive activity and the need to find an appropriate level of stress in learning processes.
\end{abstract}

Key words: classification ability, cortisol, hormonal change, stress in learning process

\section{Introduction}

According to an analysis of the 6th and 7th science curriculum, the proportion of the classification inquiry is extremely low (Kim et al., 2007). Scientific inquiry elements are limited to controlling variables and operating given experiment procedures despite it is well known that science inquiry activities promote students' interest and motivation (Wong et al., 2008). Also, the importance of classification in the science class has been underscored even though classification is a main element of scientific inquiry. What are the reasons school science does not carry out classification activity? Can we say that memorizing classification keys improve students' ability of classification?

Cortisol hormone increases in the wake of physical or emotional responses to stress (Izawa et al, 2008). According to James-Lange theory, physiological psychology, if you know changes in physiological state of the subject in advance, you can predict changes in emotion and behaviors (Kalat, 2004). Scientific inquiry increases the cognitive load of subjects and causes the physiological changes (Kalat, 2004). The choice between giving up the task and fulfilling the task to generate knowledge is determined by physiological changes directly measured from their saliva samples. Cortisol hormone is synthesized and secreted by adrenal cortex and passes through the blood to the target organ. However, some cortisol hormones can exist freely in saliva while most cortisol hormones are combined with albumin. Free-form cortisol hormones can move from blood to saliva easily because the molecular weight is light in freeform 〈Figure 1〉. Therefore, salivary cortisol hormone change in students can be considered as an indicator of students' classification ability quotient. According to this point of view, researchers set up the purpose of this study to

\footnotetext{
*Corresponding author: Yong-Ju Kwon(kwonyj@knue.ac.kr)

** Received on 30 June 2012, Accepted on 26 July 2012

***This work was supported by the National Research Foundation of Korea(NRF) grant funded by the Korea government(MEST) (No. KRF 2012-0005148)
} 
investigate relation between the classification ability quotient and physiological change of middle school students. Researchers extract subjects' saliva samples before and after the task so as to analyze the amount of the secreted hormone.

\section{Methods and Procedure}

\section{Participants}

A total of 33 middle school students who live in Gangnam-gu Seoul participated in this study in order to identify the relationship between learner's classification capability index and changes in salivary cortisol hormones. Subjects for the study must be the healthy persons who contain basic knowledge on and have enthusiasm in scientific inquiry. To meet these criteria, students having experiences and interests in scientific inquiry participated in this study voluntarily.

\section{Procedures}

The task presented to students is the feathers classification task. Students scatter twenty species' bird feathers, varied in properties such as shape and color, and then classify these feathers on their own (figure 2).

Total experiment was conducted about fiftyfive minutes and classification activity took thirty minutes. The first five minutes were used for introduction on the task. For next ten minutes, $2 \mathrm{ml}$ of saliva samples were collected from each student. After sampling, students

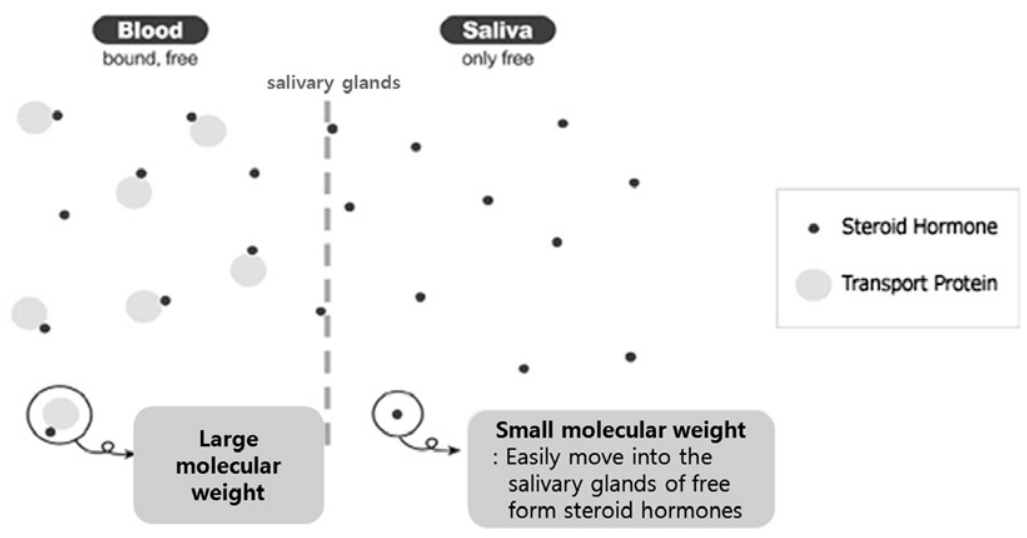

Fig. 1 Two forms of cortisol hormone in blood and saliva

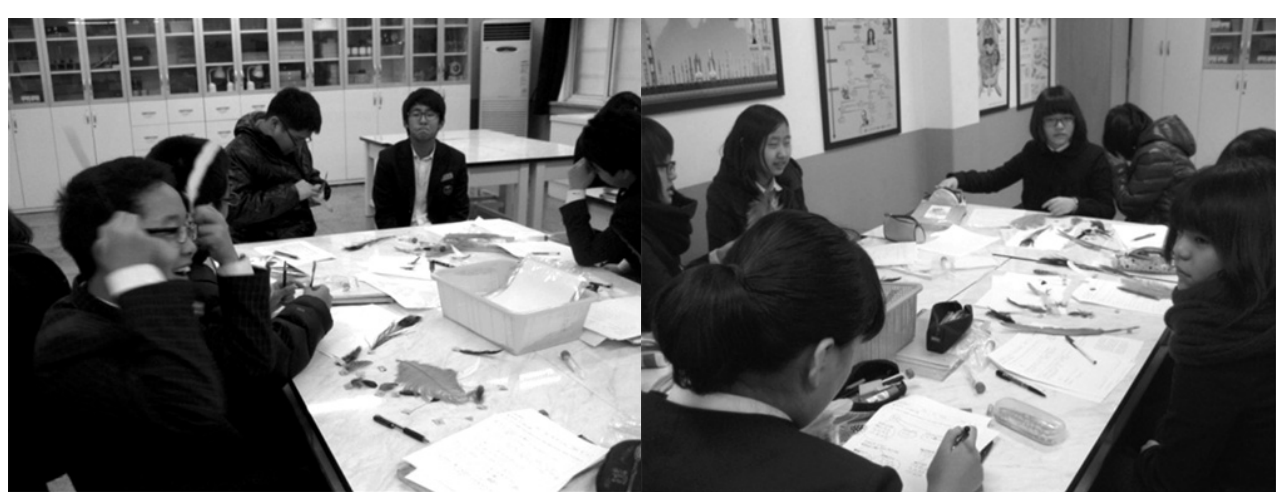

Fig. 2 Classification activity scene 
conducted the feathers classification task for thirty minutes. Students recorded the results of classification, which could be used for analyzing students' CQ, classification ability quotient $\langle$ Fig. $3\rangle$. When the classification activity was over, $2 \mathrm{ml}$ of saliva samples were extracted again from participants. Brief outline of the procedures is shown at Figure 4.

\section{Analysis}

\section{A. Analysis by type of classification assessment elements.}

Classification ability assessment tool was used to assess students' results. Criterion of the classification (CR), Accuracy of criterion (AC),
Level of the classification (LC) and Type of the classification (TC) comprise of classification ability assessment tool (Kwon et al., 2007). Experts in the field of scientific thinking and biology education analyzed the result data and inter-analyzer coincidence was 0.94 .

\section{B. Classification ability quotient (CQ)}

Kwon et al. (2007) presented the way of calculating classification ability quotient (CQ) to quantify a subject's classification ability. Researchers could acquire qualitative and quantitative information from result of classification ability quotient. Categorization of qualitative information can suggest categorization type of student whether multiple categorization

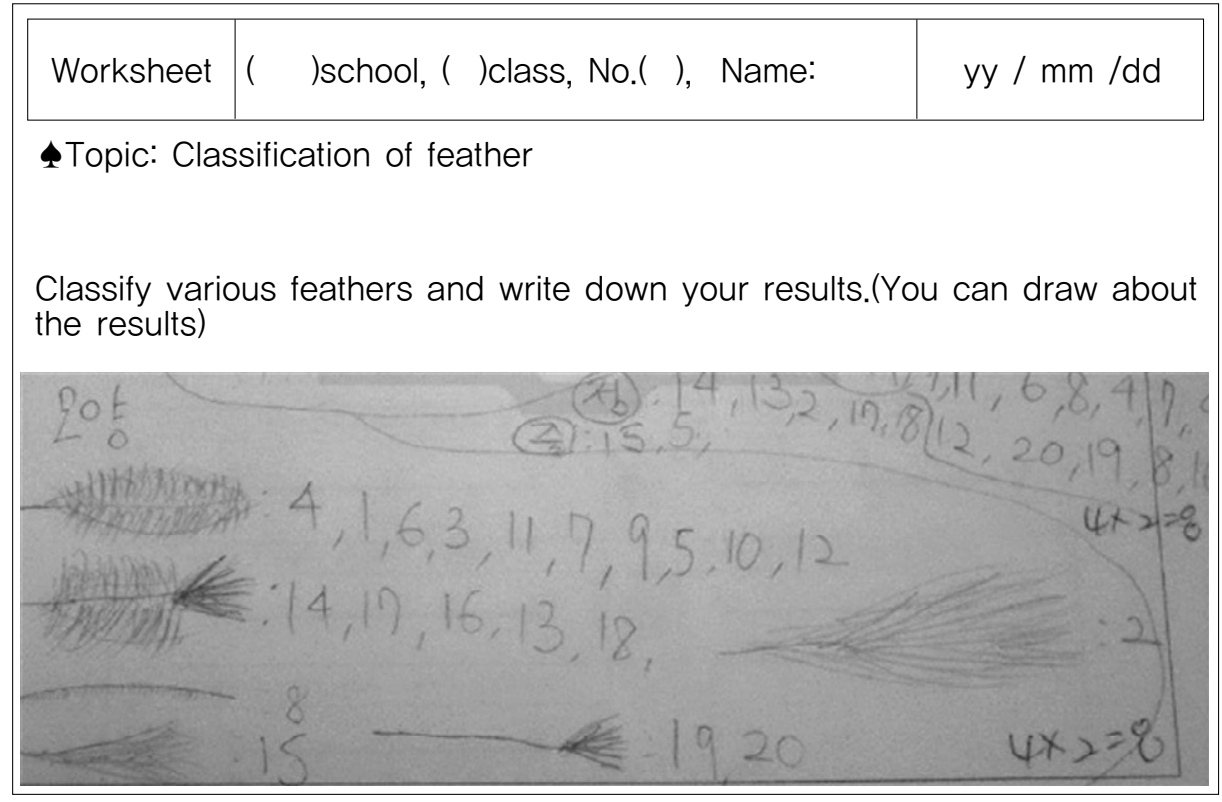

Fig. 3 Student report

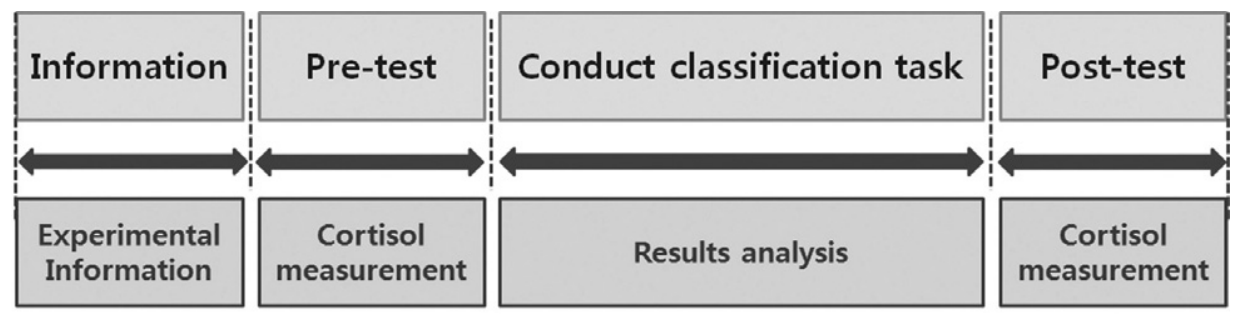

Fig. 4 Procedure of data collection 
or dual categorization. Also, hierarchical information can provide recognition level of relation among character of objects. Furthermore, researchers could quantify classification ability of each student during paper and pencil test for classification.

\section{Measurement of Change in Physiological Status of the Learner}

To find out the physiological change of learners according to the execution of given assignment, SHA (Salivary Hormone Analysis) was used. About $2 \mathrm{mlof}$ saliva sample was collected over two-time period using a test tube including an absorbent swab. The saliva sample absorbed in the swab was separated in an exclusive tube and then was analyzed. This study used salivary hormone analyzing devices and tubes for sample collecting made by Carlcam Pharmaceutical Co., LTD in order to measure the amount of cortisol hormone secretion 〈Figure 6〉. The reference range of salivary cortisol hormone is 13-24 nM when getting up in the morning, 5$10 \mathrm{nM}$ at about $11 \mathrm{AM}, 3-8 \mathrm{nM}$ in the afternoon around $4 \mathrm{PM}$, and 1-4 nM before going to bed.

\section{Results and Arguments}

\section{Analysis of classification knowledge generated by students}

A total of 136 keys of classification were generated. Two-dimensional classification keys found within the classification objects (simple

$$
\mathrm{CQ}=\Sigma\{\mathrm{CRn} \times \mathrm{LEn} \times \Sigma(\mathrm{TC} \times \mathrm{ACn})\}
$$

Classification ability quotient $=1^{\text {st }}$ classification unit $\{$ Criterion of the clssification $\times$ Level of the classification $\times\left(1^{\text {st }}\right.$ Type of the classification $\times 1^{\text {st }}$ Accuracy of criterion $)+$ $\cdots+\mathrm{n}-\mathrm{st}\}+\cdots+\mathrm{N}$-st classification unit (taxon)

(CQ : Classification ability quotient, CR : Criterion of the classification, LE : Level of the classification, TC : Type of the classification, AC : Accuracy of criterion, $\mathrm{N}: \mathrm{N}-\mathrm{st}$ classification unit (taxon), $\mathrm{n}: \mathrm{n}$-st criterion)

Fig. 5 Calculation of classification ability quotient $(C Q)$ in a single group

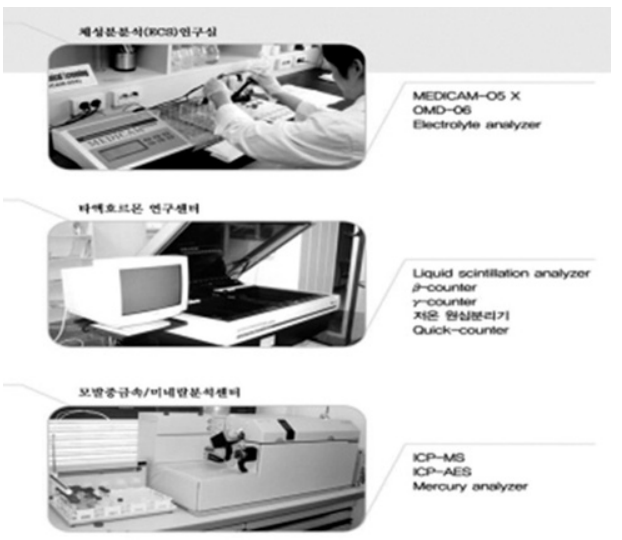

A

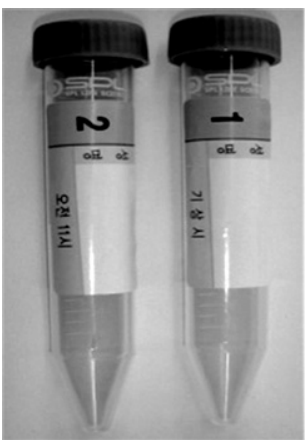

B

Fig. 6 Salivary hormone analyzing devices (A) and exclusive tube (B) 
classification, classification points of unit classification: 3) appeared 72 times (52.9). Diverse classification keys found within the classification objects (complex classification, classification points of unit classification: 4) appeared 48 times (35.3\%). 15(or 11\%). Two-dimensional classification keys out of the classification objects (simple classification, classification points of unit classification: 1) were shown 15 times (11.0\%). Diverse classification keys out of the classification objects (complex classification, classification points of unit classification: 2) were shown just once 〈Table 1〉.

\section{Table 1}

Frequency and ratio of classification points of unit classification

\begin{tabular}{ccc}
\hline \hline $\begin{array}{c}\text { Classification } \\
\text { points of } \\
\text { unit classification }\end{array}$ & Frequency & Ratio (\%) \\
\hline 1 & 15 & 11.0 \\
\hline 2 & 1 & 0.73 \\
\hline 3 & 72 & 52.9 \\
\hline 4 & 48 & 35.3 \\
\hline
\end{tabular}

In terms of precision, classification keys which are objective, qualitative and related to items of classification objects had the largest portion of 87.5\% (119 times). Classification keys which are related to items of classification objects but are inaccurate (accuracy point: 1) took 11.7\% (16 times). Classification keys which are objective, quantitative and related to items of classification objects (accuracy point: 3) took only 0.7\% (1 time). Lastly, classification keys irrelevant to the objects of classification (accuracy point: 0) were not shown 〈Table 2〉. The average number of classification keys produced per person was four.

Learners mainly used visual, simple and qualitative keys for classifications even though this task constrained no limits on the classification methods. Also, the fact that no keys exceeded two dimensions shows the immaturity of learners in classification due to the lack of experiences in systematic classification activity.
The absence of awareness for an objective also might limit the activeness of classification and the generation of classification keys.

\section{Table 2}

Frequency and ration of the accuracy of classification standards

\begin{tabular}{ccc}
\hline \hline $\begin{array}{c}\text { The accuracy of } \\
\text { classification } \\
\text { standards }\end{array}$ & Frequency & Ratio (\%) \\
\hline 0 & 0 & 0 \\
\hline 1 & 16 & 11.7 \\
\hline 2 & 119 & 87.5 \\
\hline 3 & 1 & 0.73 \\
\hline
\end{tabular}

\section{Distribution of Classification ability Quotient (CQ)}

The classification knowledge made by subjects was qualitative, comprised of sentence structures. It is hard to compare them with the amount of salivary cortisol hormone secretion. Accordingly, qualitative sentence structures were converted into quantitative data with the relative classification ability quotient equation developed by Kwon et al. (2007). The classification ability quotient of each subject showed various figures from points 6 to points 44. Just few of students, however, recorded high points. Even 5 students exhibited point 1 . These results can be interpreted that students had a difficulty to classify and that classification education was not carried out systematically. Figure 7 shows the graph of classification ability quotient of each student.

The classification ability quotient for each learner was widely distributed. On the other hand, overall high figure was not revealed. This is because most students performed onedimensional classification and used just few keys for classification. Thirty three learners and one learner showed a big difference in classification ability quotient (Figure 7). This rises from the fact that learner 33 employed more keys for classification compared to learnerlalthough both learners did a simple one-dimensional classification. 


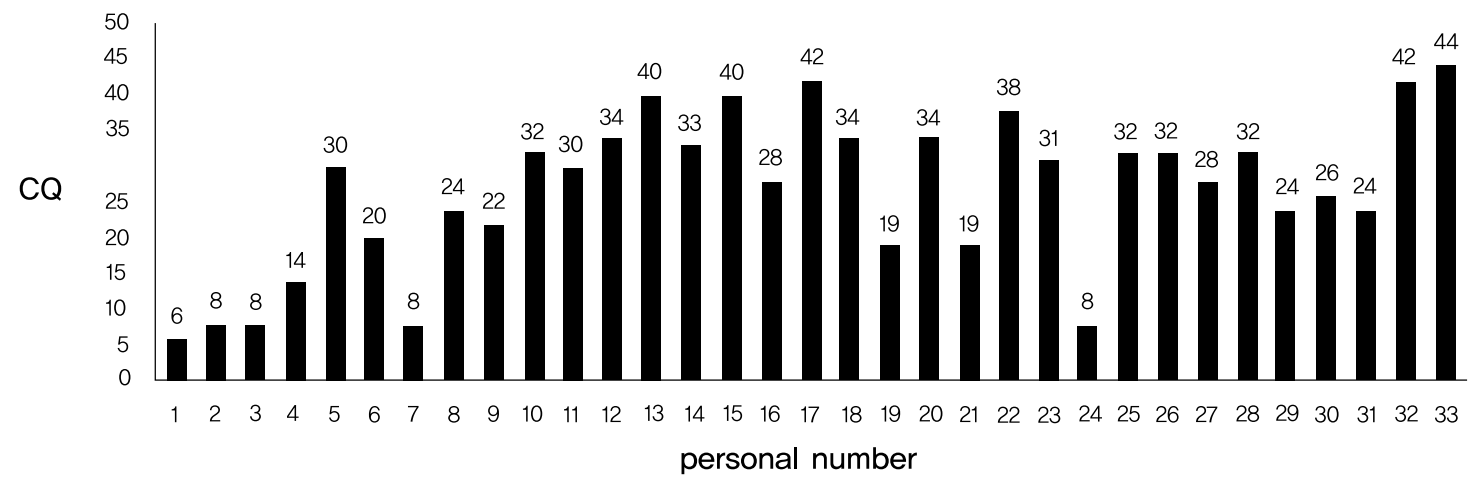

Fig. 7 Classification ability Quotient of each person

\section{Analysis on the change in Classification ability Quotient and amount of salivary cortisol hormone secretion}

The overall change in the amount of average salivary cortisol hormone secretion of learners within the group participating in the research is shown in Table 3. Initially, the amount of salivary cortisol hormone secretion was $17.3 \pm$
$7.55 \mathrm{nM}$ but after the classification activity, the amount of salivary cortisol hormone secretion decreased to $14.9 \pm 5.20 \mathrm{nM}$ and the difference was meaningfully high $(p<0.001)$.

The following shows the relationship between the classification ability quotient and the change in the amount of salivary cortisol hormone secretion 〈Figure 8$\rangle$.

The classification ability quotient showed a

\section{Table 3}

Amount of average salivary cortisol hormone secretion of learners within the group participating in the research $(N=33)$

\begin{tabular}{ccccc}
\hline \hline Hormone & Time & Density(nM) & t & P \\
\hline \multirow{2}{*}{ Cortisol } & Before & $17.3 \pm 7.55 \mathrm{nM}$ & \multirow{2}{*}{5.001} & .000 \\
\cline { 2 - 3 } & After & $14.9 \pm 5.20 \mathrm{nM}$ & & \\
\hline
\end{tabular}

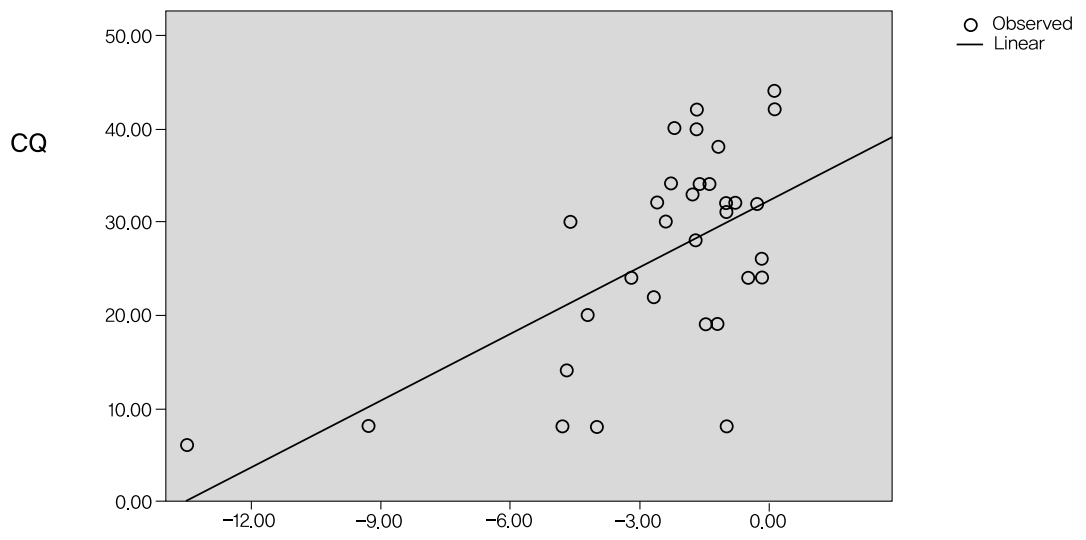

Change of salivary cortisol hormone secretion

Fig. 8 Relationship between the classification ability quotient and the change in the amount of salivary cortisol hormone secretion 
positive correlation with the change in the amount of salivary cortisol hormone secretion in a meaningful degree $\left(R^{2}=0.192, p<0.01\right)$. It can be said that cognitive burden stimulated pituitary gland and the secretion of salivary cortisol hormone was induced while students carried out feather classification task. The change in the amount of salivary cortisol hormone, therefore, can be used as a quotient to measure the classification ability. Though it is not known if the salivary cortisol hormone is secreted due to the classification activity which induces a high level of cognitive burden or due to the secretion of salivary cortisol hormone which induces the awakening of the brain and it is not known which one of the two comes first, we can acknowledge that the classification ability and the secretion of salivary cortisol hormone is closely related.

\section{Conclusion and Educational Implications}

This research seeks to find the relationship between the classification ability quotient of middle school students in the process of feathers classification task and the change in the amount of salivary cortisol hormone secretion. Thirty three students participated in this study conducted classification activity in a free and open environment and before and after executing their assignments, $2 \mathrm{ml}$ of saliva of each student was collected in order to analyze the amount of hormone. A correlation analysis was performed to find out the relativeness between the classification ability and the change in the amount of salivary cortisol hormone secretion.

\section{Conclusion}

Researchers could suggest some conclusions based on result of this study. First of all, the classification knowledge produced by students in second grade of middle school showed a variety in terms of standard of classification but remained only at the one-dimensional simple classification level and hardly showed twodimensional classification level. Also, in terms of standard of classification, they seldom used quantitative standards but mostly showed visual and qualitative ones. This indicates how much the biology education in Korea puts an emphasis more on memorizing 'the classification standards' given by the textbooks rather than on the classification ability of students in elementary schools and middle schools. Also, middle school students showed a lower tendency for classification activities compared to when they were in elementary schools and most of them were concentrated on classifying objects with one standard and did not go on to the next stage as noted by preceding researches (Lee et al. 2008).

Second, the execution of the classification assignment brought about physiological change of students. Correlation exists between the classification ability quotient and the change in the amount of salivary cortisol hormone secretion. As a result of an active performance, the amount of salivary cortisol hormone secretion decreased overall among learners. Experience-oriented activities for students decrease the level of stress. If preceding researches only compared the educational accomplishment level as an indicator of the class effects, this research directly measures the physiological change and thus will be a basic data for objective and quantitative analysis to confirm the class effects.

Varied factors can impose stress to learners. Thus we cannot exclude the environmental factors when thinking of stress-inducing factors in learners. As a result of this research, we can assume that when the amount of initial salivary cortisol hormone secretion is high, the learneroriented activities decreased the amount of salivary cortisol hormone secretion and thus the stress of learners decreased. However, this research is about the most basic scientific 
activity, classification. There are limitations such results may be due to the characteristic of this particular assignment. It is hard to predict that the results of all activities will be identical with those of this study. If research of various subjects and environments are added, this physiological method can be a diagnostic and prescriptive measurement model on teaching and learning effectiveness.

Third, the classification ability quotient and the change in the amount of salivary cortisol hormone secretion showed a positive correlation. If the amount of the salivary cortisol hormone secretion reasonably increases compared to the initial stages of the research, learners will have high level of pertinacity on assignments compared to the initial stages. Likewise, the performance ability on the classification assignment will be enhanced. On the other hand, the classification ability quotient is low in the case of students with low level of hormone change and low amount of initial salivary cortisol hormone secretion. This means that the salivary cortisol hormone secretion has a feedback relationship with classification ability. High level of stress from the initial stage leads to give up the assignment and as a result, the stress of those students gets lessened and the salivary cortisol hormone secretion is decreased. When the pertinacity for the assignment is strengthened, the salivary cortisol hormone secretion is increased. Referring to preceding research, the positive feeling at the initial stage of scientific research plays a positive role in carrying out the research and has a close relationship to knowledge production (Slavkin 2001). On the other hand, high salivary cortisol hormone density at the initial stage of scientific research plays a negative role in executing scientific assignment. While there are various factors affecting scientific ability, this study result shows that the physiological change can be objectively measured by analyzing the effect of salivary cortisol hormone secreted during scientific activity.

\section{Educational Implications}

The result of this study could implicate various fields related scientific classification activity. First of all, the analysis of classification type will become a direct background data to design strategies of the classification education. It is also expected classification analysis tool utilized in this study can be integrated to systematic classification lessons in science education. If we offer a proper classification strategy at the right time and design a learning strategy ranging from simple classification to complex, from qualitative standards to quantitative standards, from visual classification standards to operational classification standards, we can expect advancement in classification ability of students (Sim \& Chung 1996). In order to enhance classification ability, we need to design an effective classification lesson plan that accords with the classification objects and standard. Teachers need to make efforts for students' quality classification. Also, the objective of classification lessons should not end at merely separating subjects and grouping them but should enhance the acquisition of classification knowledge and cultivate basic scientific.

Second, the method of this study will serve as the guideline for measuring the internal state more accurately in a physiological level rather than through behavioral changes or self-surveys used in the existing researches. Unlike precedent researches that measured learning performance by the level of accomplishment based on behavioral or external results, this study showed the possibility of direct measurement of learning effects by means of physiological changes of learners.

Third, salivary cortisol hormone shows learners'level of correspondence on stress and implies the importance of the development of a classification task that can enhance the performance ability by inducing the right amount of stress when the classification task 
stimulate learners' learning process. If we can find the right level of salivary cortisol hormone density, learners will be able to maintain the right level and motive themselves. The salivary cortisol hormone density within learners can enhance learners' ability. When the amount of salivary cortisol hormone secretion is too high or too low, there may be difficulties in performing assignments. However, the right level of salivary cortisol hormone can actually enhance the assignment performance and assignment pertinacity. Therefore, if a strategic lesson is carried out to the extent which students can maintain the right amount of salivary cortisol hormone density, the students will be able to stimulate scientific thinking and enhance problem-solving skills. Moreover, students will engage themselves voluntarily in science classes.

\section{References}

Izawa, S., Sugaya, N., Shirotsuki, K., Yamada, K., Ogawa, N., Ouchi, Y., Nagano, Y., Suzuki, K. \& Nomura, S. (2008). Salivary dehydroepiandrosterone secretion in response to acute psychological stress and its correlations with biological and psychological changes. Biological psychology, 79(3), 294-298.

Kalat, J. W. (2004). Biological psychology. Thomson Learning.

Kim, H., Park, B., \& Lee, B. (2007). Analysis of the Basic Inquiry Process in Korean Science
Textbook: Focused on Classification, Prediction and Reasoning. The Korean Society of Elementary Science Education, 26(5), 499-508.

Kwon, Y., Lee, J., Shin, D. \& Jeong, J. (2007). Development of the Classification Ability Quotient Equation through the Analysis of Science Teachers Classification Knowledge Generated in the Pollen Classification Task. Secondary Education Research, 55(3), 21-43.

Lee, J., Ha, M. \& Cha, H. (2008). Difference in Elementary Student Behaviors according to the Material Types Provided as Classifying Leaves. Journal of Korean Elementary Science Education, 27(3), 287-295.

Sim, J. \& Chung, W. (1996). The Teaching Effect Based on Psychological Hierarchy of the Middle School Students in the Botanical Classification Concepts. The Korean Journal of Biology Education, 24(2), 199-209.

Slavkin, M. (2001). Problem Solving in the Natural Sciences and Early Adolescent Girls' Gender Roles and Self-Esteem: A Qualitative and Quantitative Analysis from an Ecological Perspective. Journal of Women and Minorities in Science and Engineering, 7(3), 241-268.

Wong, S., Hodson, D., Kwan, J. \& Yung, B. H. W. (2008). Turning Crisis into Opportunity: Enhancing Student-Teachers' Understanding of Nature of Science and Scientific Inquiry through a Case Study of the Scientific Research in Severe Acute Respiratory Syndrome. International Journal of Science Education, 30(11), 1417-1439. 